\title{
High power laser semiconductor interactions: A Monte Carlo study for silicon
}

\author{
K. Yeom, H. Jiang, ${ }^{\text {a) }}$ and J. Singh \\ Department of Electrical Engineering and Computer Science, The University of Michigan, Ann Arbor, \\ Michigan 48109-2122
}

(Received 15 July 1996; accepted for publication 3 November 1996)

\begin{abstract}
In this article, we use Monte Carlo methods to study the interaction of high power laser pulses with electrons in the conduction band of semiconductors. The laser field is represented by a sinusoidal electric field which tends to cause an oscillatory motion in the electrons. The scattering of electrons from the lattice force the electrons to lose phase coherence with the field. The approach is applied to silicon. We use the approach to examine the carrier energy distribution and material breakdown due to the transfer of energy from the laser to the electrons followed by impact ionization. The impact ionization coefficient, $\alpha$, and its dependence on the laser frequency and field strength is examined and compared to the values in a dc field. In general, the ac value is smaller than the dc value, but at low frequencies and high field strengths, the ac impact ionization coefficient approaches the dc value at the same rms field value. The importance of collisions in the energy transfer process is elucidated. (C) 1997 American Institute of Physics. [S0021-8979(97)00304-6]
\end{abstract}

\section{INTRODUCTION}

Impact ionization related breakdown of semiconductors plays a very important role in microelectronics. This phenomenon limits the high power performance of transistors since at high applied biases, the current in the device increases uncontrollably due to carrier multiplication. This phenomenon is also exploited for the design of avalanche photodetectors to provide high gain. Knowledge of the impact ionization coefficient is therefore of great importance.

The experimental measurement of the impact ionization coefficient is rather difficult due to the difficulty of maintaining uniform fields and avoiding current instabilities. As a result, there is considerable uncertainty in the impact ionization coefficients ( $\alpha$ for electrons, $\beta$ for holes) even in widely used semiconductors such as $\mathrm{Si}, \mathrm{GaAs}$, and InP. One technique that has recently been successfully applied to this problem is the use of short-pulse, high-power lasers. ${ }^{1-4}$ It is seen in these experiments (which are based on pump-probe techniques) that as the power of a laser pulse is increased (using shorter pulse widths), electron-hole $(e-h)$ plasma formation occurs signifying the onset of breakdown. In Ref. 1, this approach has been used to obtain impact ionization in $\mathrm{SiO}_{2}$ a material which has a very high breakdown field. If the photon energy is smaller than the band gap, electron generation through band to band transitions is absent in first order and the dominant carrier generation process is through impact ionization. If photons have an energy larger than the band gap, band to band transitions (whose strength is defined through absorption coefficient) compete with impact ionization induced $e-h$ generation. In Ref. 4, plasma formation has been observed in Si using above band gap photons. While this work has shed some light on breakdown in $\mathrm{Si}$ at fields as high as $10 \mathrm{MV} / \mathrm{cm}$, it is not entirely clear whether the breakdown is due to impact ionization or band to band photon

\footnotetext{
a) Also at Department of Physics, The University of Michigan, Ann Arbor, Michigan 48109-1120.
}

absorption. It is expected that with advances in short pulsehigh power lasers, it will be possible to get below band gap laser sources that can be applied to $\mathrm{Si}$ studies.

The ability of short pulse width, high-power lasers pulses to deliver very large energies to electrons in the conduction band of materials can also be exploited for novel devices. In order to fully exploit the potential of this technique, it is important to develop an understanding of how short laser pulses interact with electrons in semiconductors. In particular, it is important to answer the following questions: (i) How is the impact ionization produced by a laser field related to the dc impact ionization? (ii) What is the dependence of the energy transferred to the carriers on the frequency and power (rms field strength) of the laser? (iii) What is the nature of the nonequilibrium distribution function that describes the carriers in the laser field and how is it related to the distribution function in the dc field?

The general problem of laser-charged carrier interaction for the conduction electrons in semiconductors is a complex one. In a quasiclassical formulation (in the spirit of the Drude-Zener model) one can think of the laser field to be described by an oscillating electric field with the free carriers responding to this field. Studies of free carrier absorption in $\mathrm{Si}$ suggest that this formalism is quite reasonable for photon wavelengths upto $\sim 3 \mu \mathrm{m}{ }^{5}$ The problem is made complex due to the collisions that the carriers suffer as they respond to the field. In absence of the collisions, the electrons will simply gain and lose energy from the ac field. However, as a result of the collisions, the electrons do not simply follow the field but start to move in random directions. Also for high enough fields, impact ionization can occur in which an electron in the conduction band knocks out another electron from the valence band creating an $e-h$ pair. To describe the complications as realistically as possible, we exploit the Monte Carlo method for carrier transport which has been widely used to describe transport under dc and ac fields. Recently, we have used this approach to examine laser induced breakdown. ${ }^{6}$ In this article, we carry out a Monte Carlo study 
of impact ionization caused by a high-frequency electric field (present in a laser pulse) in silicon. The study examines the dependence of impact ionization coefficient for electrons on the frequency, field strength, and the duration of the optical pulse.

In Sec. II, we discuss the formalism used for our studies. In Sec. 3, we present our results. Conclusions are given in Sec. 4.

\section{FORMALISM}

A general formalism for describing the interaction of a laser (or dc) field with electrons in a material consists of the following ingredients:

(1) An appropriate description for the band structure of the material. This is needed not only to describe the transport, but also to obtain the threshold energy for impact ionization.

(2) A description of the scattering processes, including a proper model for phonon scattering and impact ionization.

(3) A description of carrier transport so that macroscopic quantities such as $\alpha$ can be obtained.

In Sec. II, we will describe the approach used for each of these three components. Our article will focus on silicon although the approach can be readily applied to other materials. Since at present there are no experimental results on laser-silicon interactions with subband gap energy photons, we will use a simple model to address the problem. More sophisticated theories can be used later if they are warranted by experimental findings. However, the model we choose should provide a good description of dc phenomena such as velocity-field results and the impact ionization coefficients. As noted below, the model used gives accurate dc results.

\section{A. Bandstructure for electron transport}

Experimental and theoretical studies in silicon have shown that the electron initiated impact ionization coefficient $(\alpha)$ is larger than hole initiated impact ionization $(\beta)$. At low fields $(\sim 200 \mathrm{kV} / \mathrm{cm})$, the ratio $\alpha / \beta$ is $\sim 10$. At higher fields, it decreases but $\alpha$ remains larger than $\beta$ upto the highest fields where experiments are available. For this reason, we will only focus on electron transport in the conduction band. Silicon is an indirect band gap material with the conduction band edge being described by six valleys. The bottom of the conduction band in Si occurs at $2 \pi / a(0.85,0,0)$ and five other equivalent points along the $\Gamma X$ direction, where $a$ is the lattice constant of silicon $(a=5.43 \AA)$. The six valleys produced are highly anisotropic and the constant energy surfaces are ellipsoids. For low electric field $(\leqslant 50 \mathrm{kV} / \mathrm{cm})$, the inclusion of only the lowest valleys for electron transport is adequate. However, for high field transport (especially for breakdown studies), it is essential that both $L$ and $X$ valleys be included. Simple analytical expressions for the $E-k$ relationship can be used for each of these valleys since the band edges occur at different $k$ points and the valleys are not degenerate in $k$ space.

Near the band edges, indirect gap semiconductors have the form
TABLE I. Si conduction band parameters.

\begin{tabular}{ccccc}
\hline \hline Parameter & Symbol & Units & Si value & Ref. \\
\hline Nonparabolicity & $\alpha_{X}$ & $\mathrm{eV}^{-1}$ & 0.5 & 7 \\
& $\alpha_{L}$ & $\mathrm{eV}^{-1}$ & 0.3 & 8 \\
Effective mass & $m_{l X}^{*}$ & $m_{0}$ & 0.916 & 9 \\
& $m_{t X}^{*}$ & $m_{0}$ & 0.190 & 9 \\
& $m_{l L}^{*}$ & $m_{0}$ & 1.590 & 7 \\
\multirow{5}{*}{ Band edge } & $m_{t L}^{*}$ & $m_{0}$ & 0.126 & 8 \\
& $\epsilon_{X}$ & $\mathrm{eV}$ & 0 & $\ldots$ \\
\hline \hline & $\epsilon_{L}$ & $\mathrm{eV}$ & 1.05 & 8 \\
\hline
\end{tabular}

$$
E(k)=E\left(k_{0}\right)+\frac{\hbar^{2}}{2}\left[\frac{\left(k_{l}-k_{l 0}\right)^{2}}{m_{l}^{*}}+\frac{\left(k_{t}-k_{t 0}\right)^{2}}{m_{t}^{*}}\right],
$$

where $m_{l}^{*}$ and $m_{t}^{*}$ are the longitudinal and transverse effective masses and $\left(k_{l 0}, k_{t 0}\right)$ represents the bandedge $k$ point. Away from the band edges, the bands are nonparabolic in nature. Therefore, it is convenient to use a nonparabolic band structure to express the conduction band. An approximate description is given by

$$
\gamma(E)=E(1+\alpha E)=\frac{\hbar^{2}}{2}\left(\frac{k_{l}^{2}}{m_{l}^{*}}+\frac{k_{t}^{2}}{m_{t}^{*}}\right) .
$$

This expression is known to be valid for $L$ and $X$ valley conduction bands. The values of the band parameters such as nonparabolicity $(\alpha)$, effective masses $\left(m_{l}^{*}\right.$ and $m_{t}^{*}$ for longitudinal and transverse effective mass, respectively), and valley separations between $X$ and $L$ valleys are given in Table I. Values given are from Refs. 7-9.

Although the conduction band constant energy surface is ellipsoid, it is simply expressed by a form of sphere through Herring and Vogt transformation ${ }^{7}$ in the Monte Carlo simulation.

It may be noted that more sophisticated theories for a $\mathrm{Si}$ band structure have been incorporated in Monte Carlo to study impact ionization. These full band Monte Carlo approaches (see for example Ref. 10) provide a better description of the $k$-space occupation of electrons. However, as noted above, since there are no experimental studies on subband gap energy photon-Si interactions, we have chosen to use the simpler methods which give good agreement for all of the dc physical quantities of interest.

\section{B. Scattering mechanisms}

The transport of carriers in semiconductors is dominated by carriers scattering from various perturbations present in the system. In fact, without scattering, the particle will simply oscillate in the Brillouin zone creating Bloch oscillation. One can conceptually think of the particle transport as series of "free flights" in the applied field followed by scattering. The free flight trajectory of the electron is known through the equation

$$
\hbar \frac{d \mathbf{k}}{d t}=e \mathbf{F},
$$

where $\mathbf{F}$ is the electric field. The energy of the particle is simply obtained from the $E$ vs $k$ relation. 
TABLE II. Si electron phonon scattering parameters.

\begin{tabular}{lcccc}
\hline \multicolumn{1}{c}{ Parameter } & Symbol & Units & Si value & Ref. \\
\hline $\begin{array}{l}\text { Acoustic phonon } \\
\text { deformation potential }\end{array}$ & $D_{X}$ & $\mathrm{eV}$ & 9.9 & $\ldots$ \\
& $D_{L}$ & $\mathrm{eV}$ & 7.0 & $\ldots$ \\
Phonon speed & $s$ & $10^{5} \mathrm{~cm} / \mathrm{s}$ & 9.04 & 9 \\
Nonpolar optical phonon & & & & \\
deformation potential & $D_{0 X}$ & $10^{8} \mathrm{eV} / \mathrm{cm}$ & 4.51 & $\ldots$ \\
& $D_{0 L}$ & $10^{8} \mathrm{eV} / \mathrm{cm}$ & 6.80 & $\ldots$ \\
Phonon energy & $\hbar \omega_{0}$ & $\mathrm{meV}$ & 63.34 & 7 \\
\hline \hline
\end{tabular}

The most important scattering mechanism in high quality semiconductors are those related to lattice vibrations. The electron phonon scattering is described via various deformation potentials corresponding to acoustic and optical phonons. In Tables II and III, we list the important parameters which are used in our study. In addition to phonon scattering, at high fields, the carriers can suffer scattering through impact ionization as well. Also if the carrier density is high, electron-electron scattering (in the same band) can be important. Here we will study transport assuming that the initial electron density is small and carrier-carrier scattering is negligible. Of course, after the initiation of breakdown, there may be a large carrier density and $e-e$ and $e-h$ scattering may be important. By ignoring these effects, we are studying the onset of breakdown. In a later article, we will examine the importance of carrier-carrier scattering. Since a number of articles have discussed scattering processes in $\mathrm{Si}$ in detail (see Ref. 7 for a detailed list), we will only describe the model used in this study for impact ionization.

\section{Impact ionization scattering}

Impact ionization occurs due to a carrier-carrier scattering involving carriers in the conduction and valence bands. The process is mediated by Coulombic interaction. While, in principle, this scattering is simple, in practice, there is no simple $a b$ initio derivation of the scattering rate that results in an analytical model for indirect gap materials. The threshold energy for the scattering process can be derived analytically (or numerically). The threshold energy arises from the energy and momentum conservation of the particles. The

TABLE III. Si $X-X$ electron intervalley scattering parameters.

\begin{tabular}{lccc}
\hline \hline \multicolumn{1}{c}{ Parameter } & Symbol & Units & Si value \\
\hline Intervalley $(X-X)$ & & & \\
deformation potential & $D_{g 1}$ & $10^{8} \mathrm{eV} / \mathrm{cm}$ & 11.0 \\
from Ref. 9 & $D_{g 2}$ & $10^{8} \mathrm{eV} / \mathrm{cm}$ & 0.8 \\
& $D_{g 3}$ & $10^{8} \mathrm{eV} / \mathrm{cm}$ & 0.5 \\
& $D_{f 1}$ & $10^{8} \mathrm{eV} / \mathrm{cm}$ & 2.0 \\
& $D_{f 2}$ & $10^{8} \mathrm{eV} / \mathrm{cm}$ & 2.0 \\
& $D_{f 3}$ & $10^{8} \mathrm{eV} / \mathrm{cm}$ & 0.3 \\
Phonon energy & $\hbar \omega_{g 1}$ & $\mathrm{meV}$ & 62.05 \\
from Ref. 7 & $\hbar \omega_{g 2}$ & $\mathrm{meV}$ & 18.53 \\
& $\hbar \omega_{g 3}$ & $\mathrm{meV}$ & 12.06 \\
& $\hbar \omega_{f 1}$ & $\mathrm{meV}$ & 59.03 \\
& $\hbar \omega_{f 2}$ & $\mathrm{meV}$ & 47.40 \\
& $\hbar \omega_{f 3}$ & $\mathrm{meV}$ & 18.96 \\
\hline \hline
\end{tabular}

threshold energy for electron initiated impact ionization in silicon is almost the same as the band gap energy.

As noted earlier, the total scattering rate cannot be determined $a b$ initio as a simple analytical model. However, several models, including the Keldysh model, ${ }^{11,12}$ the Thoma model, ${ }^{13,14}$ and the Cartier model, ${ }^{15}$ have been proposed to give a good description of the scattering rate.

Here we use the model derived by Cartier et al. ${ }^{15}$ by fitting Monte Carlo results to experimental data. The impact ionization rate is given by

$$
R_{\text {impact }}=\sum_{i=1}^{3} \theta\left(E-E_{t h}^{(i)}\right) P^{(i)}\left(\frac{E-E_{t h}^{(i)}}{E_{t h}^{(i)}}\right)^{2},
$$

where $E_{t h}^{(i)}=1.2,1.8$, and $3.45 \mathrm{eV}$. The parameter $P^{(i)}$ are scaled in order to give a good fit of experimental results (for dc impact ionization) for the band structure and scattering rates used in this work. It may be noted that in Ref. 16 a model for impact ionization is used which gives an expression based on the conduction and valence band density of states that can be used in a simple manner in Monte Carlo simulations. The resulting rates are found to have a form similar to those obtained from the Cartier model.

\section{Transport formalism}

The formalism used to study the carrier transport is based on the Monte Carlo method. The electron Monte Carlo program is relatively simple and is based on several published works [see Ref. 6]. The usual dc Monte Carlo method is extended to include the laser field effects by using a sinusoidal electric field. ${ }^{5}$

To extend the study to include the response of the electrons to short pulse, high power lasers, we use the following equation to represent the intercollisional free flight

$$
\frac{d \mathbf{p}}{d t}=q \mathbf{F} \sin (\omega t)
$$

where $\mathbf{p}$ is a momentum of the electron. Equation (5) is then integrated to give the change in momentum during the free flight

$$
\mathbf{p}(t+\Delta t)-\mathbf{p}(t)=\frac{q \mathbf{F}}{\omega}\{\cos [\omega t]-\cos [\omega(t+\Delta t)]\},
$$

where $t$ is the time before the free flight and $\Delta t$ is the length of the free flight.

\section{RESULTS}

Before applying the Monte Carlo approach to examine the laser-electron interactions, we have chosen the parameters $P$ for impact ionization to fit experimental results. The results showing a comparison of the theoretical values and experimental values taken from the literature ${ }^{17-20}$ are shown in Fig. 1. The calculated values show a reasonable fit to the experimental data.

It is useful to consider the influence of scattering of carriers when a laser field is impinging on the sample. As dis- 


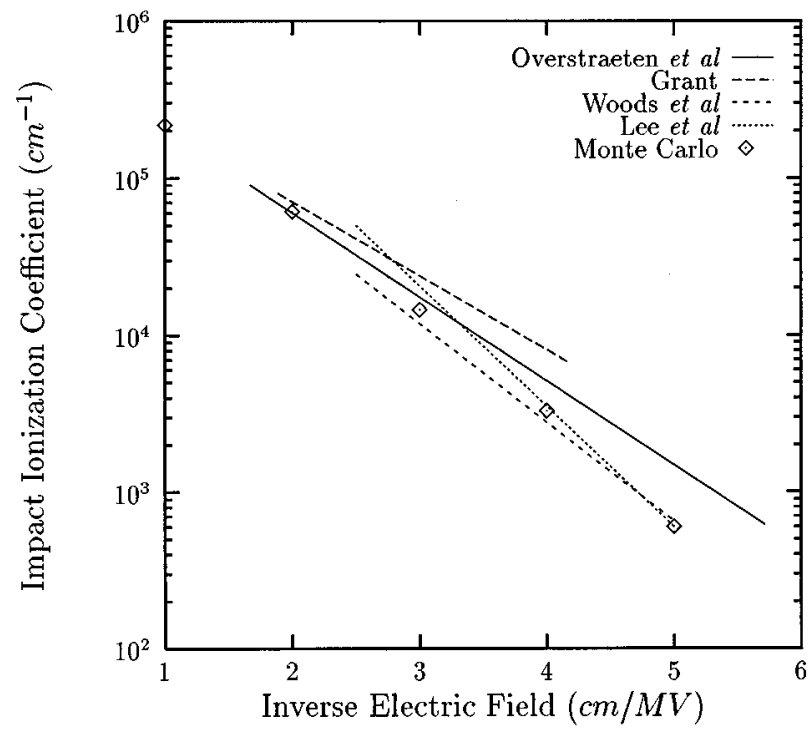

FIG. 1. Calculated impact ionization coefficient using Monte Carlo simulations. Also shown are experimental results.

cussed in Ref. 5, in the absence of scattering, in an ac field, the electron energy oscillates and the mean energy is simply given by

$$
\langle E\rangle=e^{2} F_{0}^{2} / 4 m^{*} \omega^{2},
$$

where $m^{*}$ is the carrier effective mass along the polarization direction.

It is found that if this equation is used to calculate the carrier energy for laser fields that produce breakdown, the energy value is much smaller than the band gap energy needed for breakdown. Thus, experimentally, the laser is able to deliver a much higher energy to the carriers than what is expected in absence of scattering. In fact, scattering is the key to understanding how carriers gain energy from the field. In the presence of scattering, the electron can gain energies much larger than that given by the equation above if the scattering rates is larger than the laser field frequency.

To understand the energy transfer to the electrons, and its field strength and frequency dependence, it is important to note that as the electron energy increases, the scattering rates increase. At high frequencies and low rms electric fields, the electron can follow the field without scattering for several cycles. As a result, the electron energy gain is close to that given by Eq. (7). However, if the rms field strength increases, the scattering rate also increases and the electron energy starts to approach values close to those for dc fields with strengths equal to the laser rms value.

In Figs. 2 and 3, we show the average electron energy as a function of time for ac and dc case at relatively low field and high field. The results in Fig. 2 are for a frequency of 0 (dc case) and $10^{14} \mathrm{~Hz}$ and a field of $1.0 \mathrm{MV} / \mathrm{cm}$. We can see that the average energy gained for the dc case is much larger than that for the ac case. Of course, the influence of scattering is not entirely negligible since the ac value of $\sim 0.6 \mathrm{eV}$ is larger than what we expect from Eq. (7) (i.e., a value of only $0.0044 \mathrm{eV})$.

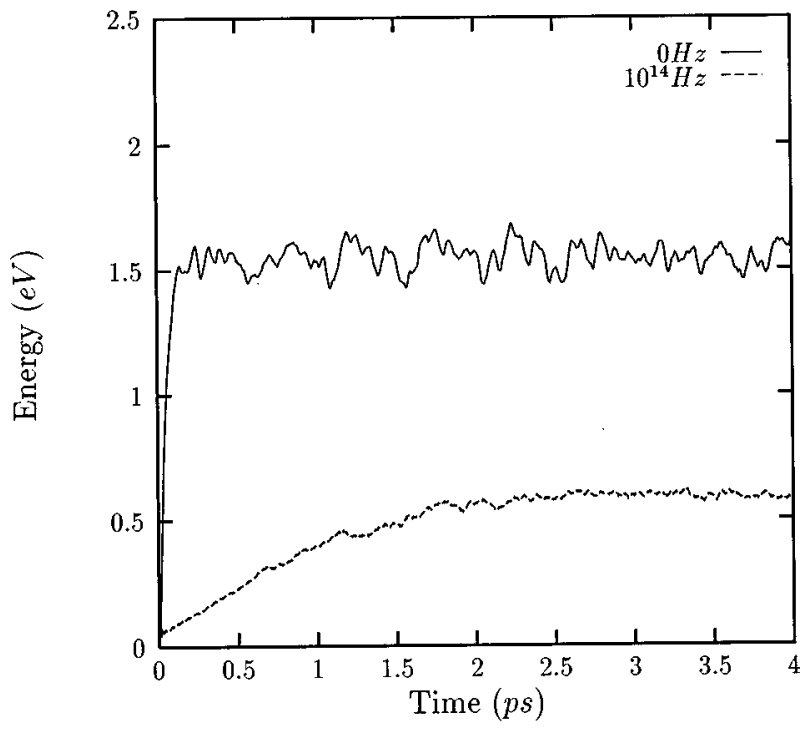

FIG. 2. Average electron energy as a function of time for an ac and dc field turned on at time zero. The dc field is equal to the rms value of the ac field which is $1 \mathrm{MV} / \mathrm{cm}$. The ac field frequency is $10^{14} \mathrm{~Hz}$.

In Fig. 3, we show the results for a rms field of 10 $\mathrm{MV} / \mathrm{cm}$. We see now that the average energies for the ac and dc cases are much closer. This can be expected since for the higher energy transferred to the carriers, the scattering rates in the semiconductor are higher and the electrons can suffer more collisions in a single cycle of the laser field.

The values of the average energy of carriers is useful but does not present a complete picture of how impact ionization will occur under dc and ac fields. This is because the impact ionization process involves a threshold energy for initiation and is primarily due to the tails in the electron distribution function. It is thus essential to examine the nonequilibrium distribution function for the carriers. We show these results in Figs. 4 and 5.

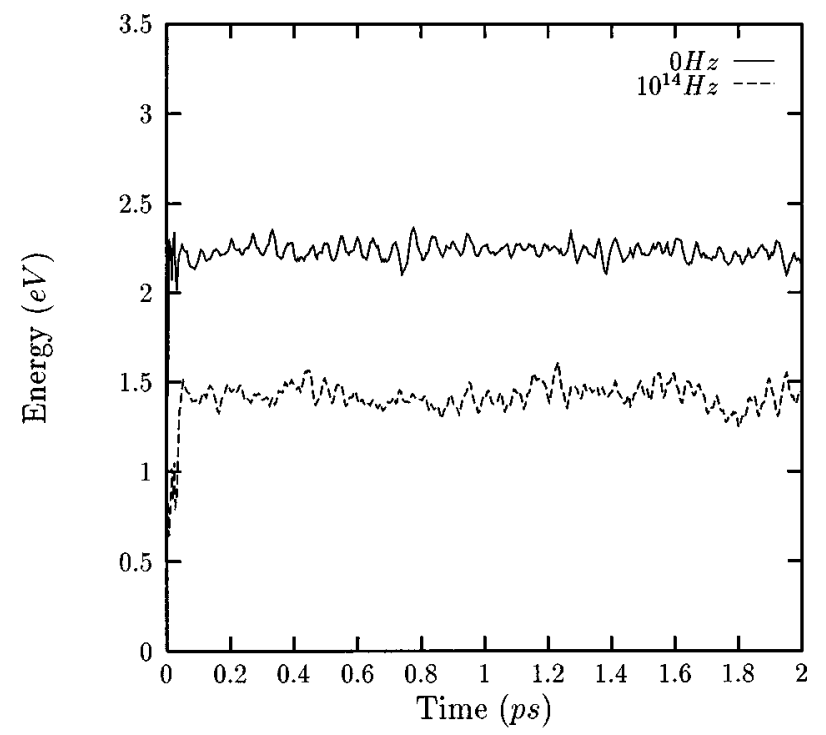

FIG. 3. Average electron energy as a function of time for an ac and de field turned on at time zero. The dc field is equal to the rms value of the ac field, which is $10 \mathrm{MV} / \mathrm{cm}$. The ac field frequency is $10^{14} \mathrm{~Hz}$. 


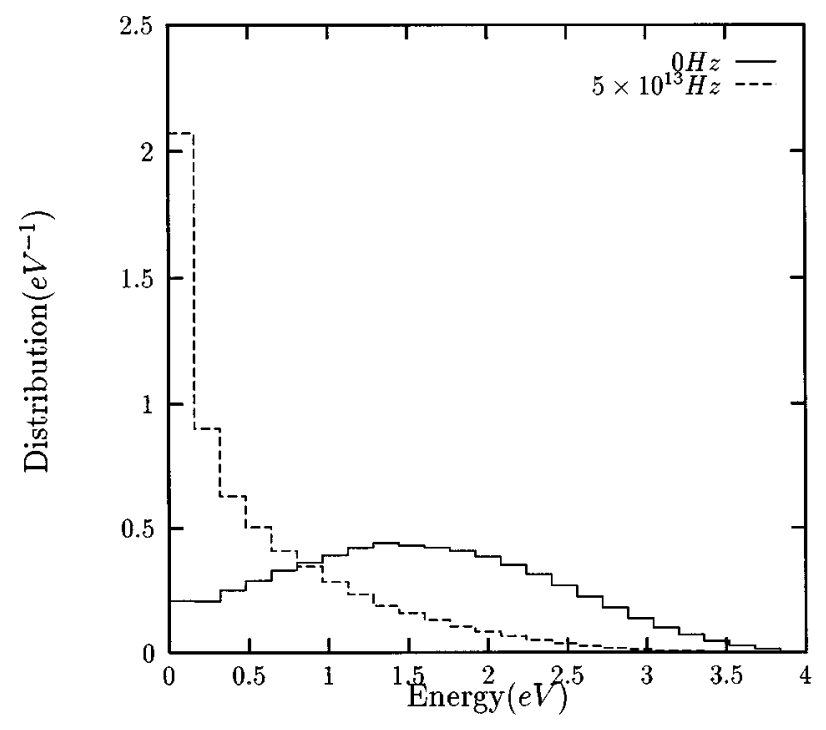

FIG. 4. Normalized distribution function for electrons in Si conduction band for ac and dc field. The rms field is $1 \mathrm{MV} / \mathrm{cm}$ and the ac frequency is $5 \times 10^{13} \mathrm{~Hz}$.

In Fig. 4, we show the normalized carrier distribution function for an ac field with an rms value of $1 \mathrm{MV} / \mathrm{cm}$ and a frequency of $5 \times 10^{13} \mathrm{~Hz}$. The carrier distribution is compared to a dc case with the dc field equal to the rms value. As expected, we see that the dc distribution function has a much higher value at high energies. This is because in the ac case the field reverses its direction before the carriers have had a chance to reach a steady state dc like energy distribution. To reach the steady state dc like distribution, the carriers must scatter several times during the field cycle.

In Fig. 5, we show the two distribution functions for the same frequency but for a field with an rms value of $10 \mathrm{MV} /$ $\mathrm{cm}$. In this figure, we see that the two distribution functions are much closer to each other. The distribution function for

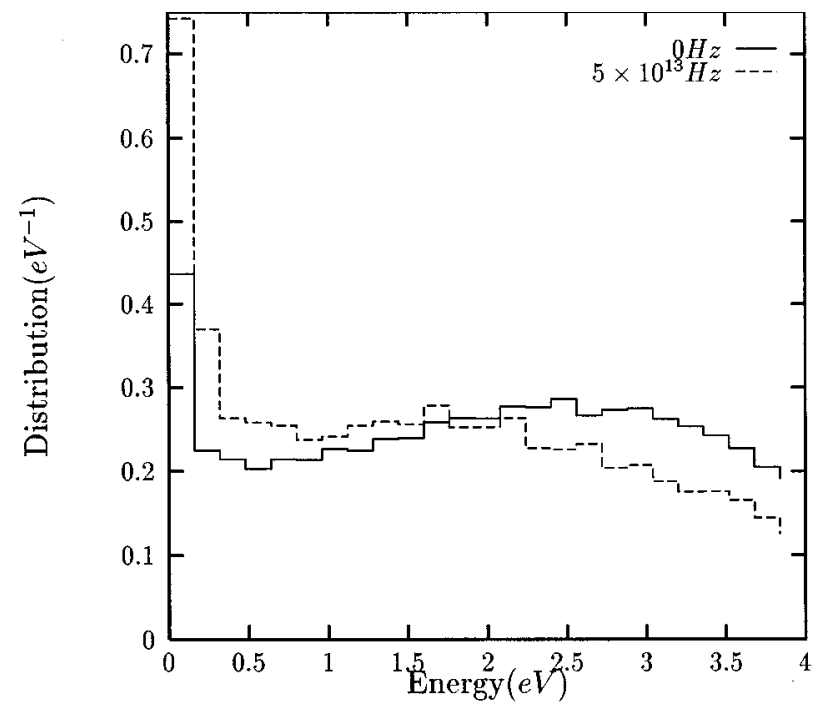

FIG. 5. Normalized distribution function for electrons in Si conduction band for ac and dc field. The rms field is $10 \mathrm{MV} / \mathrm{cm}$ and the ac frequency is $5 \times 10^{13} \mathrm{~Hz}$.

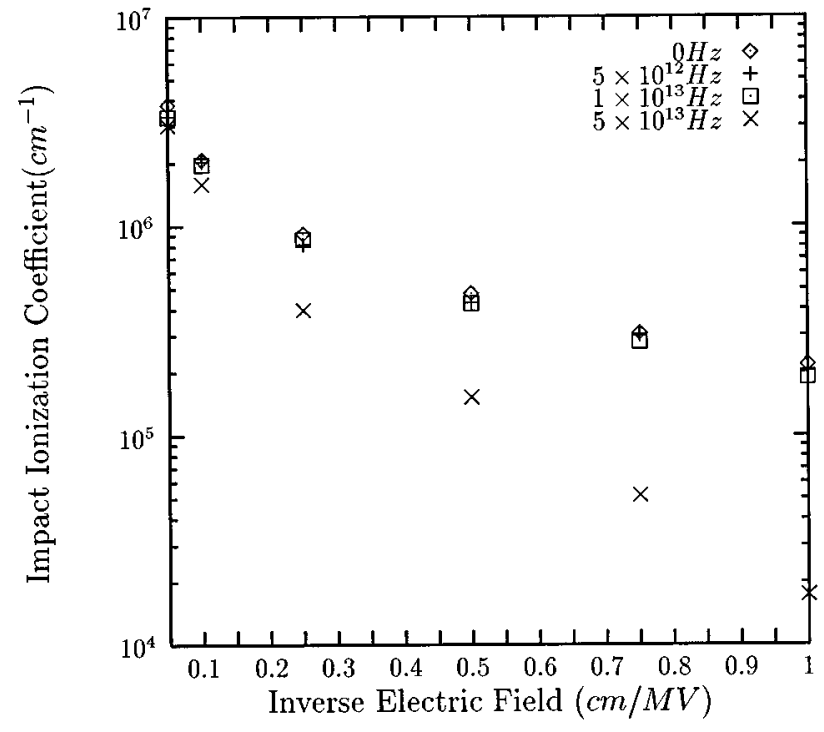

FIG. 6. Field strength dependence of impact ionization for a laser pulse and a dc field. For the laser field, the values are rms values.

the laser field has an extended tail similar to the case for the dc field.

The results shown suggest the impact ionization coefficients calculated for the ac and dc cases will be such that the ac value will be lower than the dc value but the difference will decrease for lower frequencies and for higher rms field values. This is indeed the case as shown in Figs. 6 and 7. It may be noted that the impact ionization coefficient is normally plotted in units of length inverse. However, for the ac case, the computer simulations give the results in time inverse units. We convert out results by using the drift velocity calculated for the carriers under a dc field with value equal to the rms field.

In Fig. 6, we show the frequency and field strength dependence of the impact ionization. The dc value is shown for

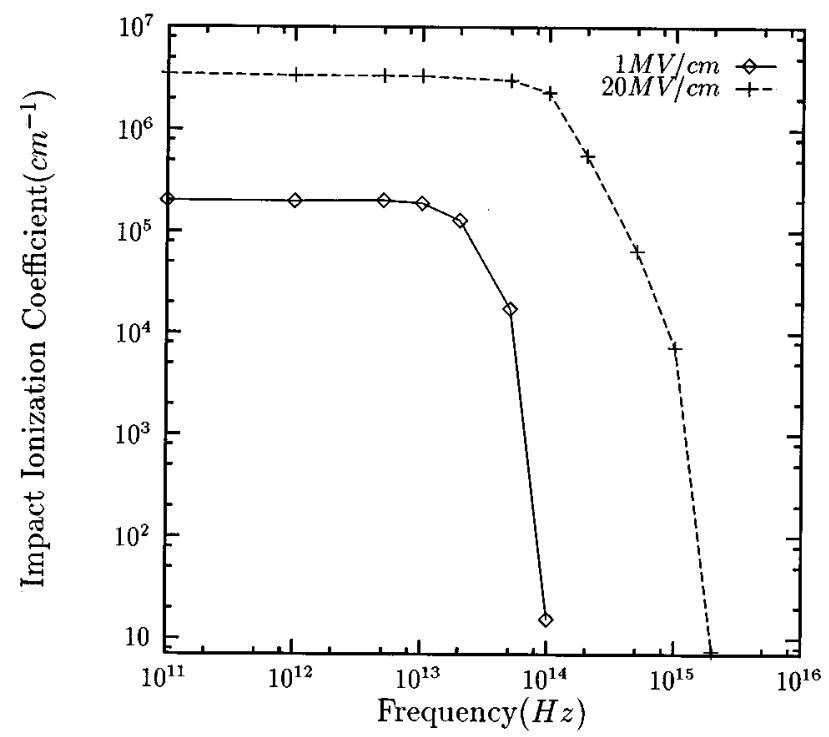

FIG. 7. Frequency dependence of the impact ionization coefficient in silicon for $\mathrm{rms}$ fields of 1 and $20 \mathrm{MV} / \mathrm{cm}$. 
comparison. We see that at low fields, the ac and dc values depart as the frequency increases to $5 \times 10^{13} \mathrm{~Hz}$. However, for higher fields, the ac and dc values remain quite close to each other. The ac and dc values are indistinguishable from each other for field strengths with rms values approaching 20 $\mathrm{MV} / \mathrm{cm}$.

In Fig. 7, we show the frequency dependence of the impact ionization coefficient for a $10 \mathrm{ps}$ pulse with a rms field of 1.0 and $20.0 \mathrm{MV} / \mathrm{cm}$. We see that the ac and $\mathrm{dc}$ values depart from each other at a frequency of $\sim 2 \times 10^{13} \mathrm{~Hz}$ for the $1.0 \mathrm{MV} / \mathrm{cm}$ case and at $\sim 2 \times 10^{14} \mathrm{~Hz}$ for the 20.0 $\mathrm{MV} / \mathrm{cm}$ case.

From our simulations, we get a scaling law for the turning frequency at which the ac and dc impact ionization coefficients start to depart

$$
\frac{\omega_{\text {turning }}}{F_{\text {rms }}}=\text { constant }
$$

for field $F_{\mathrm{rms}}>1 \mathrm{MV} / \mathrm{cm}$. It has been suggested that the rms breakdown field corresponds to the dc breakdown field, at least in ionic solids, by the relationship ${ }^{3}$

$$
F_{t h}^{\mathrm{rms}}(\omega)=F_{t h}^{\mathrm{dc}}\left(1+\omega^{2} \tau^{2}\right)^{1 / 2},
$$

where $\tau$ is the collision time. There is considerable ambiguity in $\tau$ since the collision time has a strong dependence on field strength. Using the collision time at the ac breakdown field, as calculated by Monte Carlo, we have found that the ac breakdown field as calculated by Eq. (9) does not agree with the breakdown field as determined by the ac Monte Carlo simulation. Thus, it appears that the assumptions leading to Eq. (9) are too simplistic.

\section{CONCLUSIONS}

In this article, we have carried out Monte Carlo computer simulations to study the interaction of a laser field with the electrons in a semiconductor. We have examined how the energy is transferred from the laser field to the electron gas. In particular, we have examined the importance of scattering in the energy transfer process. We find that if the number of collisions during a single cycle of the laser field is small, the carriers average energy remains much smaller than the value calculated for a dc field with the same rms value. However, if the frequency decreases or the field strength increases so that the number of collisions during a cycle is large, the ac and dc values approach each other. As an application, we have studied the impact ionization coefficient, $\alpha$, for electrons in silicon as a function of the field frequency and the rms value of the field. For fields oscillating at frequencies much below the inverse of the carrier scattering rate, the impact ionization coefficient is found to have the same value as in the dc case with the rms field replacing the dc value. At higher frequencies, the impact ionization rate decreases. The dependence of $\alpha$ on field frequency and rms field strength has been described via a simple scaling equation which is applicable at $\mathrm{rms}$ fields above $1 \mathrm{MV} / \mathrm{cm}$. This equation allows us to predict the laser power at which the ac and dc impact ionization coefficients start to differ from each other.

\section{ACKNOWLEDGMENT}

This work was funded by the U.S. Army URI program (DAAL03-92-G-0109) by the U.S. Air Force (Grant No. AFOSR-91-0349).

${ }^{1}$ D. Du, X. Liu, G. Korn, J. Squier, and G. Mourou, Appl. Phys. Lett. 64, 3071 (1994)

${ }^{2}$ E. Yablonovitch and N. Bloembergen, Phys. Rev. Lett. 29, 907 (1972).

${ }^{3}$ N. Bloembergen, IEEE J. Quantum Electron. QE-10, 375 (1974).

${ }^{4}$ P. P. Pronko, P. A. VanRompay, R. K. Singh, F. Qian, D. Du, and X. Liu, Mater. Res. Soc. Symp. Proc. 397, 45 (1996).

${ }^{5}$ W. Spitzer and H. Y. Fan, Phys. Rev. 108, 268 (1957).

${ }^{6}$ B. Kochman, K. Yeom, and J. Singh, Appl. Phys. Lett. 68, 1936 (1996).

${ }^{7}$ C. Jacoboni and L. Reggiani, Rev. Mod. Phys. 55, 645 (1983).

${ }^{8}$ M. V. Fischetti, IEEE Trans. Electron Devices 38, 634 (1991).

${ }^{9} \mathrm{~K}$. Hess, Advanced Theory of Semiconductor Devices (Prentice Hall, Englewood Cliffs, NJ, 1988).

${ }^{10}$ Y. Kamakura, H. Mizuno, M. Yamaji, M. Morifuji, K. Tanaguchi, C. Hamaguchi, T. Kunikiyo, and M. Takenaka, J. Appl. Phys. 75, 3500 (1994).

${ }^{11}$ L. V. Keldysh, Sov. Phys. JETP 10, 509 (1960).

${ }^{12}$ A. R. Beattie, Semicond. Sci. Technol. 3, 48 (1988).

${ }^{13}$ R. Thoma, H. J. Peifer, W. L. Engl, W. Quade, R. Brunetti, and C. Jacoboni, J. Appl. Phys. 69, 789 (1991).

${ }^{14}$ R. Thoma, H. J. Peifer, and W. L. Engl, J. Appl. Phys. 69, 2300 (1991).

${ }^{15}$ E. Cartier, M. Fischetti, E. Eklund, and F. McFeely, Appl. Phys. Lett. 62, 3339 (1993).

${ }^{16}$ N. Sano and A. Yoshi, J. Appl. Phys. 77, 2020 (1995).

${ }^{17}$ C. A. Lee, R. A. Logan, R. L. Bartdorf, J. J. Kleimack, and W. Wieggmann, Phys. Rev. A 134, 761 (1964).

${ }^{18}$ R. Van Overstraeten and H. De Man, Solid-State Electron. 13, 583 (1970).

${ }^{19}$ W. N. Grant, Solid-State Electron. 16, 1189 (1973).

${ }^{20}$ M. H. Woods, W. C. Johnson, and M. A. Lampert, Solid-State Electron. 16, 381 (1973). 